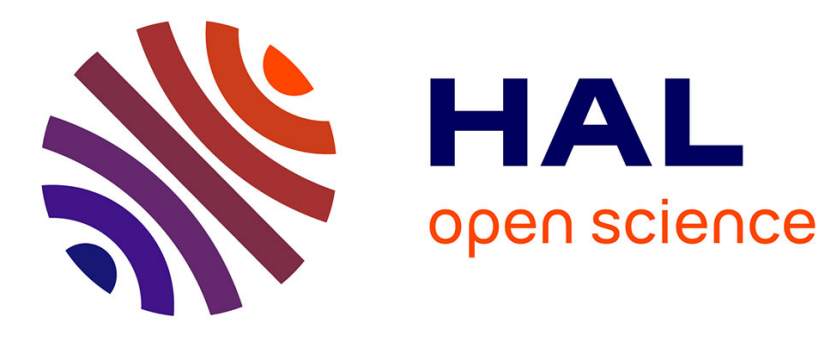

\title{
Spectroscopic decomposition of astronomical multispectral images using B-splines
}

Hassan Mortada, Vincent Mazet, Charles Soussen, Christophe Collet

\section{To cite this version:}

Hassan Mortada, Vincent Mazet, Charles Soussen, Christophe Collet. Spectroscopic decomposition of astronomical multispectral images using B-splines. Whispers, Sep 2018, Amsterdam, Netherlands. 10.1109/WHISPERS.2018.8747032 . hal-01871674

\section{HAL Id: hal-01871674 \\ https://hal.science/hal-01871674}

Submitted on 21 Oct 2021

HAL is a multi-disciplinary open access archive for the deposit and dissemination of scientific research documents, whether they are published or not. The documents may come from teaching and research institutions in France or abroad, or from public or private research centers.
L'archive ouverte pluridisciplinaire HAL, est destinée au dépôt et à la diffusion de documents scientifiques de niveau recherche, publiés ou non, émanant des établissements d'enseignement et de recherche français ou étrangers, des laboratoires publics ou privés. 


\title{
SPECTROSCOPIC DECOMPOSITION OF ASTRONOMICAL MULTISPECTRAL IMAGES USING B-SPLINES
}

\author{
Hassan Mortada ${ }^{1}$, Vincent Mazet ${ }^{1}$, Charles Soussen ${ }^{2}$ and Christophe Collet $^{1}$ \\ ${ }^{1}$ ICube, Université de Strasbourg - CNRS, Illkirch, France \\ ${ }^{2}$ Laboratoire des Signaux et Systèmes, CentraleSupélec-CNRS-Univ. Paris-Saclay, Gif-sur-Yvette, France \\ E-mail: \{mortada, vincent.mazet, c.collet\}@unistra.fr, charles.soussen@ centralesupelec.fr
}

\begin{abstract}
This paper addresses the estimation of the kinematic structures composing a galaxy from a multispectral image. Each structure corresponds to an emission line embedded in the spectra of the image: the goal is to estimate the amplitude, shift, and shape of the peaks assuming that these parameters evolve slowly between two adjacent pixels. These parameters are modeled as B-spline surfaces to favor their smooth spatial evolution. The B-spline parameterization allows one to describe the multispectral image using a reduced number of parameters. Results on synthetic and real data validate the proposed method.
\end{abstract}

Index Terms - Galaxy kinematics, sequence of signals, smooth evolution, splines.

\section{INTRODUCTION}

Galaxies and intergalactic gas are in motion due to inner physics and the Universe expansion [1]. The emission lines in their spectra are shifted, proportionally to their apparent speed: this is the well-known Doppler effect, also known as redshift. A multispectral observation of an object shows different redshifts in each pixel (or spectrum). Besides, emission lines may be embedded many times in a spectrum if different gas speeds are observed in the line of sight.

The goal of this paper is to estimate the redshifts in a multispectral observation of a galaxy, as it is considered in [13]. This is equivalent to estimate the characteristics of the emission lines (called peaks in the sequel), i.e., their amplitude, spectral shift and shape. It is also needed to follow the peaks through the observation to be able to infer the underlying kinematic structures. The problem is challenging because the peaks change from one spectrum to another and are very correlated.

A common assumption in astronomy is to model the peaks as a Gaussian [2] or Moffat [4] function. We also consider that the peak characteristics evolve slowly from one pixel to its neighbors (see Fig. 1) : the set of peaks showing a slow spatial

This work was funded by the DSIM project (grant ANR-14-CE27-0005).

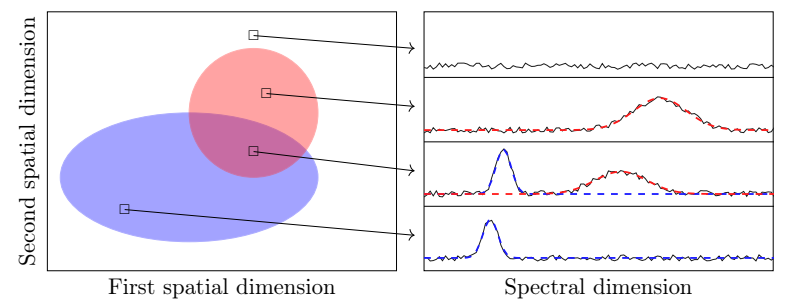

Fig. 1: Left: a synthetic example of two overlapping structures (red and blue). Right: spectra at four spatial positions (in black) and the peaks to estimate (with colors indicating their structure).

evolution is called a structure and a galaxy may have different structures.

Galaxy kinematic in multispectral images has been studied in $[3,5,6]$. In [2], a Bayesian approach was proposed that also deals with the deconvolution of the image; however, this method considers only one kinematic structure. Bouché et al. [7] proposed to use a parametric model to fit the galaxy kinematic, but the shift evolution was constrained to be linear. On the contrary, spectral-spatial classification $[8,9]$ considers only the classification task of each pixel without estimating the peak characteristics in each pixel. Besides, the problem can also be seen as convolutive source separation problem [10], where the spectra and peaks are respectively associated with the mixtures and sources. However, most of the classical approaches make strong assumptions on the sources, such as their statistical independence and decorrelation, which is not valid in a real-application world such as in astronomy. Other works, such as [11-13], are adapted to spectroscopic signals, but they do not suppose the slow evolution of the peak characteristics and cannot be applied to the detection of spatially connected structures in an image. On the contrary, some works [14] are adapted to multispectral images but use an instantaneous source separation model which cannot model the spectral shift and the shape evolution.

We propose to model the spatial evolution of the peak characteristics through the image as B-spline surfaces to satisfy the slow evolution of the characteristics from one pixel to its 
neighbors. In consequence, the estimation of the characteristics is replaced by the estimation of the B-spline control points, yielding a significant reduction in the number of unknowns. The generative model is presented in section 2 . The inverse problem is then addressed as a non-linear least squares optimization problem and the proposed optimization strategy is detailed in section 3 . Results on synthetic and real data are presented in section 4 .

The notations used in this paper are as follows: bold and lowercase variables correspond to vectors; bold and uppercase variables correspond to matrices; the $j$ th column of a matrix $M$ is denoted as $M_{: j}$, and the notation $M_{:, 1: j}$ selects the first $j$ columns of $M$.

\section{PROBLEM FORMULATION}

Suppose the multispectral image has $I$ pixels. We denote $i \triangleq\left[\begin{array}{ll}u & v\end{array}\right]$ a pixel with coordinates $u$ and $v$. Each spectrum $\boldsymbol{x}_{\boldsymbol{i}}=\left[\begin{array}{lll}x_{\boldsymbol{i}}(1) & \ldots & x_{\boldsymbol{i}}(N)\end{array}\right]^{T} \in \mathbb{R}^{N}$ gathers samples at $N$ wavelengths and is the noisy sum of $J$ peaks (modeled by a parametric function $s\left(\cdot ; w_{i j}\right)$ ) shifted in $c_{i j}$ with spatially varying shape parameter $w_{i j}$ :

$$
\boldsymbol{x}_{\boldsymbol{i}}=\sum_{j=1}^{J} a_{i j} \boldsymbol{s}\left[c_{\boldsymbol{i} j} ; w_{i j}\right]+\boldsymbol{n}_{\boldsymbol{i}}
$$

where

$$
\boldsymbol{s}\left[c_{\boldsymbol{i} j} ; w_{\boldsymbol{i} j}\right]=\left[\begin{array}{lll}
s\left(1-c_{\boldsymbol{i} j} ; w_{\boldsymbol{i} j}\right) & \ldots & s\left(N-c_{\boldsymbol{i} j} ; w_{\boldsymbol{i} j}\right)
\end{array}\right]^{T}
$$

is a $N$-dimensional vector gathering the samples of the peak $j$, $a_{i j}$ is the amplitude of peak $j$ in pixel $\boldsymbol{i}$ and $\boldsymbol{n}_{\boldsymbol{i}} \in \mathbb{R}^{N}$ models the noise. The physics imposes that $a_{i j} \geq 0$ and $c_{i j} \in[1, N]$. Moreover, we impose that $w_{i j} \in\left[w_{\min }, w_{\max }\right]$ where $w_{\min }$ and $w_{\max }$ are respectively the lower and upper bounds for the shape parameter.

In order to impose a slow evolution of the parameters, their evolution is modeled by bicubic B-splines i.e., bidimensional functions defined from two cubic B-splines. A cubic B-spline is a piecewise polynomial of degree $3[15,16]$. The so-called knot vector $\boldsymbol{k}_{u}$ defined over the first spatial dimension $u$ allows us to define $P$ cubic B-spline basis functions $b_{1}(u), \ldots, b_{P}(u)$. Similarly, a knot vector $\boldsymbol{k}_{v}$ defined over the second spatial dimension $v$ allows us to define $Q$ B-spline basis functions $b_{1}(v), \ldots, b_{Q}(v)$. A bicubic B-spline basis function centered in $[u v]$ is then defined as:

$$
\beta_{p, q}(\boldsymbol{i})=b_{p}(u) b_{q}(v)
$$

The amplitudes, shifts and shape parameters of a structure $j$ are modeled as a linear combination of $M=P \times Q$ bicubic B-splines (without loss of generality, the same B-spline bases functions are used to model the parameters):

$$
\begin{aligned}
& a_{\boldsymbol{i} j}=\sum_{p=1}^{P} \sum_{q=1}^{Q} \phi_{p, q}^{j} \beta_{p, q}(\boldsymbol{i})=\boldsymbol{\beta}_{\boldsymbol{i}}^{T} \boldsymbol{\phi}_{j} \quad \forall \boldsymbol{i}, j \\
& c_{\boldsymbol{i} j}=\sum_{p=1}^{P} \sum_{q=1}^{Q} \sigma_{p, q}^{j} \beta_{p, q}(\boldsymbol{i})=\boldsymbol{\beta}_{\boldsymbol{i}}^{T} \boldsymbol{\sigma}_{j} \quad \forall \boldsymbol{i}, j \\
& w_{\boldsymbol{i} j}=\sum_{p=1}^{P} \sum_{q=1}^{Q} \psi_{p, q}^{j} \beta_{p, q}(\boldsymbol{i})=\boldsymbol{\beta}_{\boldsymbol{i}}^{T} \boldsymbol{\psi}_{j} \quad \forall \boldsymbol{i}, j
\end{aligned}
$$

where the discrete B-spline bases functions are gathered into $\boldsymbol{\beta}_{\boldsymbol{i}}^{T} \in \mathbb{R}^{M}$, and the so-called control of the amplitudes $\phi_{p, q}^{j}$, shifts $\sigma_{p, q}^{j}$ and shapes $\psi_{p, q}^{j}$ are respectively gathered into $\phi_{j}$, $\boldsymbol{\sigma}_{j}$, and $\boldsymbol{\psi}_{j}$.

Eq. (1) now writes:

$$
\boldsymbol{x}_{\boldsymbol{i}}=\sum_{j=1}^{J} \boldsymbol{\beta}_{\boldsymbol{i}}^{T} \boldsymbol{\phi}_{j} \boldsymbol{s}\left[\boldsymbol{\beta}_{\boldsymbol{i}}^{T} \boldsymbol{\sigma}_{j} ; \boldsymbol{\beta}_{\boldsymbol{i}}^{T} \boldsymbol{\psi}_{j}\right]+\boldsymbol{n}_{\boldsymbol{i}}
$$

The estimation of the peak characteristics is thus equivalent to that of the control points $\phi_{j}, \sigma_{j}$ and $\psi_{j}$. Assuming a white Gaussian noise, the maximum likelihood estimator minimizes the following criterion:

$$
\mathcal{O}(\boldsymbol{\Phi}, \boldsymbol{\Sigma}, \boldsymbol{\Psi})=\sum_{\boldsymbol{i}}\left\|\boldsymbol{x}_{\boldsymbol{i}}-\sum_{j=1}^{J} \boldsymbol{\beta}_{\boldsymbol{i}}^{T} \boldsymbol{\phi}_{j} \boldsymbol{s}\left[\boldsymbol{\beta}_{\boldsymbol{i}}^{T} \boldsymbol{\sigma}_{j} ; \boldsymbol{\beta}_{\boldsymbol{i}}^{T} \boldsymbol{\psi}_{j}\right]\right\|_{2}^{2}
$$

where $\boldsymbol{\Phi}, \boldsymbol{\Sigma}$ and $\boldsymbol{\Psi}$ respectively gather the control points of the amplitudes $\phi_{j}$, shifts $\sigma_{j}$ and shape parameters $\psi_{j}$.

Finally, (8) is minimized, yielding the following constrained non-linear least squares problem:

$\min _{\boldsymbol{\Phi}, \boldsymbol{\Sigma}, \boldsymbol{\Psi}} \mathcal{O}(\boldsymbol{\Phi}, \boldsymbol{\Sigma}, \boldsymbol{\Psi})$ such that $\mathcal{C}=\left\{\begin{array}{l}\boldsymbol{\Phi} \in \mathbb{R}_{+}^{M \times J} \\ \boldsymbol{\Sigma} \in[1, N]^{M \times J} \\ \boldsymbol{\Psi} \in\left[w_{\min }, w_{\max }\right]^{M \times J}\end{array}\right.$

\section{CONTROL POINT ESTIMATION}

Because of the non-linearity of the unknowns ( $\boldsymbol{\Sigma}$ and $\boldsymbol{\Psi}$ ), (8) is non-convex and the choice of the initial solutions for optimization solver is crucial, especially for the shift control points. We adopt an iterative strategy where the number of estimated structures is incremented by one at each iteration. In addition to the estimation of a new structure, the parameters of the previous ones are also updated at each iteration. We propose to initialize the shift control points as the wavelength of highest intensity in the average spectrum (computed by averaging all the spectra of the image); this is a simple and efficient choice for finding a potential peak.

The proposed algorithm is given in Algorithm 1. At each iteration, the number $k$ of structures to estimate is incremented 


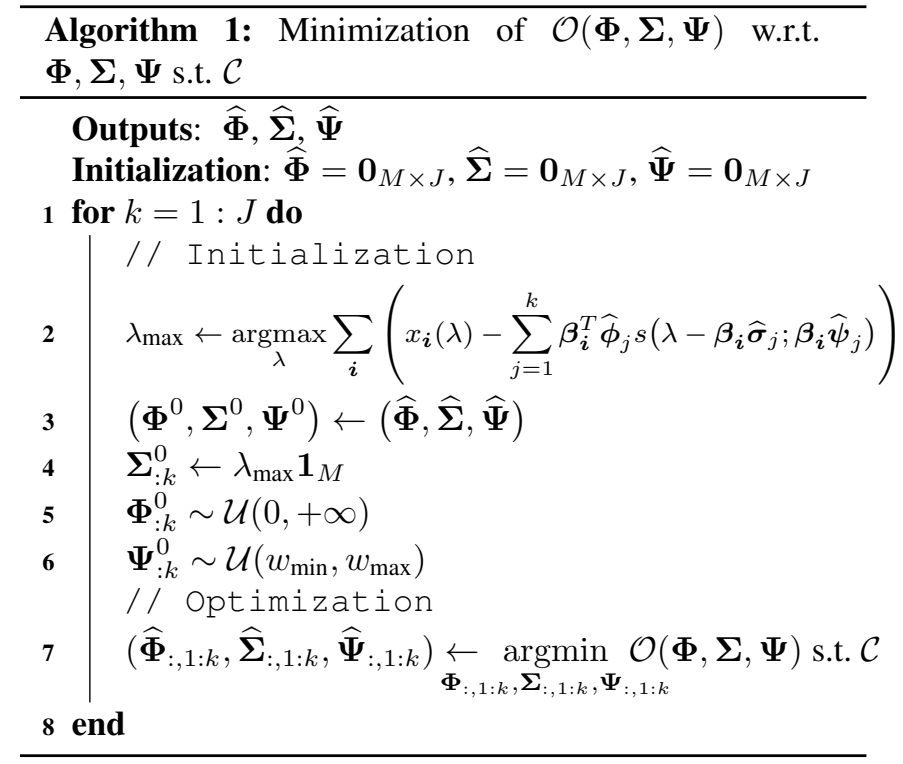

from $k=1$ to $k=J$. In other words, the optimization problem (9) is solved with respect to a subset of the parameters, namely the first $k$ columns of $\boldsymbol{\Phi}, \boldsymbol{\Sigma}$ and $\boldsymbol{\Psi}$ (line 7). A sequential quadratic programming [17] is used to solve the optimization problem. The solution found at iteration $k$ is put in the first $k$ columns of the initial solutions $\boldsymbol{\Phi}^{0}, \boldsymbol{\Sigma}^{0}$ and $\boldsymbol{\Psi}^{0}$ at iteration $k+1$ (line 3). The $k+1$ column of $\boldsymbol{\Phi}^{0}$ and $\boldsymbol{\Psi}^{0}$ is set to a random value generated following a uniform distribution $\mathcal{U}$ over the intervals in $\mathcal{C}$ (lines 5, 6). Whereas, all the elements in the $k+1$ column of $\Sigma^{0}$ are set, as said above, to the wavelength with the highest intensity in the average residual spectrum $\lambda_{\max }$ (line 4). The computation of $\lambda_{\max }$ takes place in line 2 .

\section{RESULTS}

\subsection{Synthetic Image}

To evaluate the performance of our method, we use a synthetic multispectral image of dimension $50 \times 50 \times 70$ and $J=2$ structures that overlap in both spatial and spectral domains. The two structures fill the image. The peaks are parameterized by a Gaussian function $s(\lambda ; w)=e^{-\lambda^{2} / 2 w^{2}}$. The ground truth parameters are generated using $\mathrm{B}$-spline surfaces with random control points and are displayed in Fig. 3. An additive Gaussian noise with signal-to-noise ratio ${ }^{1}$ equal to $10 \mathrm{~dB}$ is added to the image spectra. The knot vectors of the amplitudes, shifts and shapes are the same in the two spatial dimensions and are given by: $\boldsymbol{k}_{u}=\boldsymbol{k}_{v}=\left[\begin{array}{lllllllllll}1 & 1 & 1 & 1 & 13 & 26 & 38 & 50 & 50 & 50 & 50\end{array}\right]$. The first and last 4 knots are identical to allow for variable boundary conditions [18]. The results in Fig. 2 show that the multispectral image is correctly reconstructed (the mean

\footnotetext{
${ }^{1}$ Defined as 10 times the log-ratio of the mean energy of the noiseless spectra and the noise variance.
}

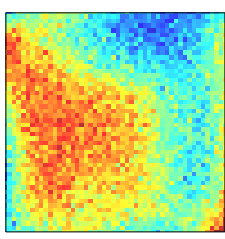

(a)

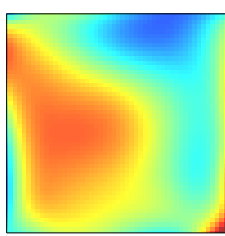

(b)

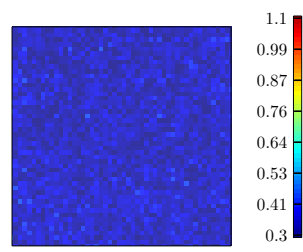

(c)
Fig. 2: The synthetic cube (a), the reconstruction computed from the estimation (b) and their absolute difference (c). The images represent multispectral images averaged along the spectral dimension. The same color dynamic is used for the three figures.

square error equals 0.18 ). Furthermore, the parameters are visually well estimated and keep a smooth evolution as expected (Fig. 3). Thanks to the B-spline modeling of the parameters, the number of unknowns is reduced from 5, 000 (the number of amplitudes, shifts and shapes for $I=50^{2}$ pixels and $J=2$ structures) to 384 . The computation time of the proposed approach is only 8 minutes (with Matlab).

\subsection{Galaxy NGC 4254}

The proposed method is applied to a real observation in the radio band (around the $\mathrm{HI}$ peak at $21 \mathrm{~cm}$ ) of the galaxy NGC 4254 [19] (Fig. 4a). The image is of dimension $140 \times 140 \times 42$. The number of structures is set to $J=2$. Surface B-splines with the following knots are used to model the shifts and shapes: $\boldsymbol{k}_{u}=\boldsymbol{k}_{v}=$ $\left[\begin{array}{lllllllllllll}1 & 1 & 1 & 1 & 24 & 47 & 70 & 93 & 116 & 140 & 140 & 140 & 140\end{array}\right]$. The amplitudes are modeled with more knots so as to fit the complex behavior of the intensities in the cube: $\boldsymbol{k}_{u}=\boldsymbol{k}_{v}=$ $\left[\begin{array}{llllllllllllllll}1 & 1 & 1 & 1 & 12 & 33 & 44 & 54 & 65 & 76 & 87 & 97 & 108 & 119 & 129 & 140\end{array}\right.$ 140140 140]. The number of unknowns is thus reduced from 39, 200 (the number of amplitudes, shifts and shapes for $I=140^{2}$ pixels and $J=2$ structures) to 706 .

Fig. $4 \mathrm{~b}$ and $4 \mathrm{c}$ show that the galaxy is well reconstructed (the mean square error equals $6.8 \cdot 10^{-7}$ ). The estimated parameters are displayed in Fig. 5. The pixels with estimated amplitudes lower than a small value (threshold) are removed from the estimation. Indeed, the first estimated structure represents the main kinematic structure of the Galaxy: two spiral arms rotating around the center, yielding a different redshift in each side of the galaxy. The estimated parameters of the second structure highlights the presence of three structures. The structure at the top is an artificial structure that was added for evaluating the performance of the method: it is a disk with a decreasing amplitude and a constant shift, as it is confirmed in the results. The structure at the left is very faint and difficult to find but the proposed method was able to find it automatically. Finally, the structure at the right is mostly a part of the main kinematic structure: the peaks in this region of the image is not really Gaussian so a second structure is needed to fit the 


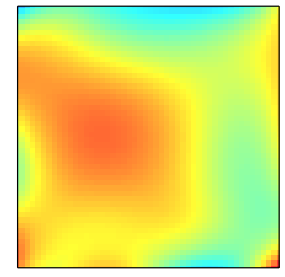

(a) $\boldsymbol{A}_{1}^{*}$

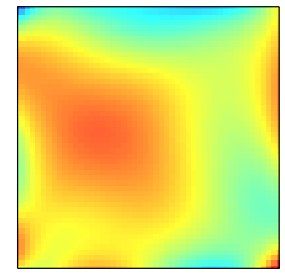

(g) $\widehat{A}_{1}$

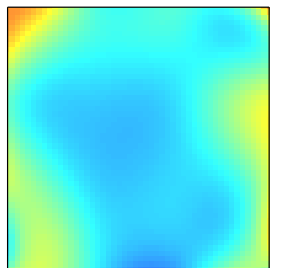

(b) $C_{1}^{*}$

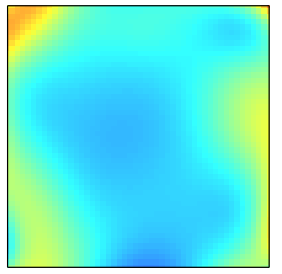

(h) $\widehat{\boldsymbol{C}}_{1}$

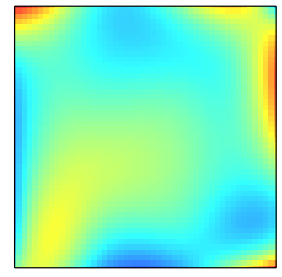

(c) $\boldsymbol{W}_{1}^{*}$

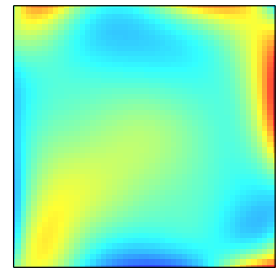

(i) $\widehat{\boldsymbol{W}}_{1}$

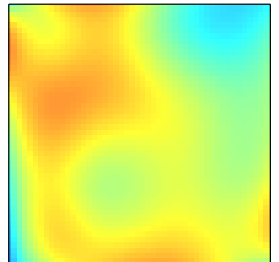

(d) $\boldsymbol{A}_{2}^{*}$

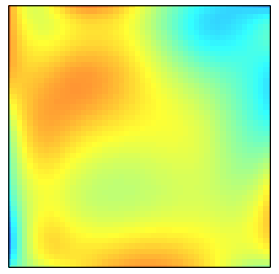

(j) $\widehat{A}_{2}$

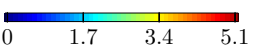

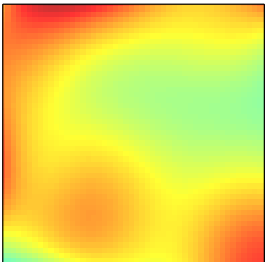

(e) $C_{2}^{*}$

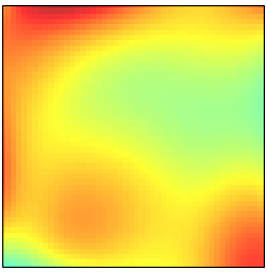

(k) $\widehat{\boldsymbol{C}}_{2}$

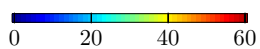

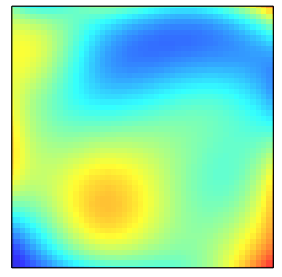

(f) $\boldsymbol{W}_{2}^{*}$

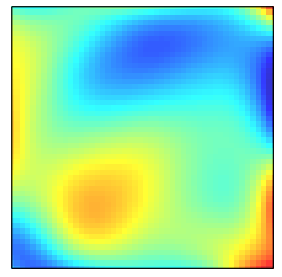

(1) $\widehat{\boldsymbol{W}}_{2}$
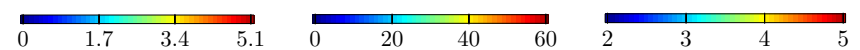

Fig. 3: (a)-(c) The ground truth of the amplitudes $\boldsymbol{A}$, shifts $\boldsymbol{C}$ and shapes $\boldsymbol{W}$ of the first structure (resp. (d)-(f) for the second structure). (g)-(i) the estimated parameter of the first structure (resp. (j)-(1) for the second structure).

peaks.

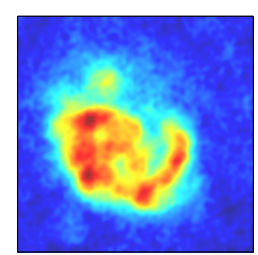

(a) $\boldsymbol{X}$

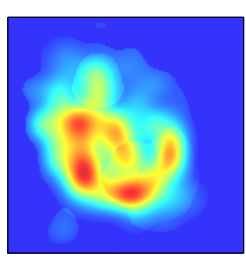

(b) $\widehat{\boldsymbol{X}}$

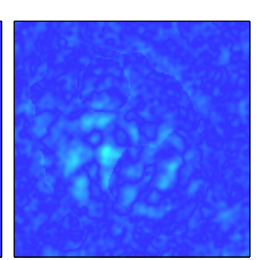

(c) $|\boldsymbol{X}-\widehat{\boldsymbol{X}}|$
Fig. 4: Real data (galaxy NGC 4254) with $J=2$ structures. (a) Averaged image of the data $\boldsymbol{X}$ along the spectral dimension. (b) Averaged image of the reconstruction $\widehat{\boldsymbol{X}}$ (after thresholding) (c) and of the residual .

\section{CONCLUSION}

This paper presents a novel method to estimate multiple kinematic structures within a galaxy multispectral image. The peak characteristics (amplitude, shift and shape) representing the structures in each spectrum are modeled by cubic B-spline surfaces to ensure their smooth spatial evolution. Besides, this modeling yields a significant reduction in the number of unknown. The problem is addressed as a constrained non-linear least squares problem and an iterative algorithm is proposed where the structures are iteratively estimated. Results on synthetic and real data show the effectiveness of the algorithm.

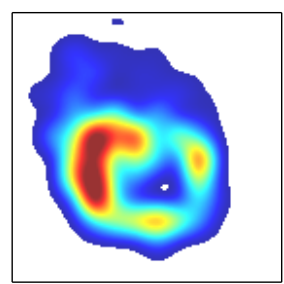

(a) $\widehat{A}_{1}$

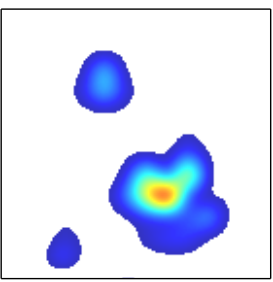

(d) $\widehat{A}_{2}$

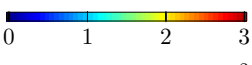

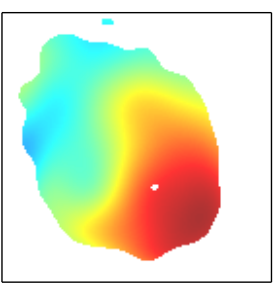

(b) $\widehat{C}_{1}$

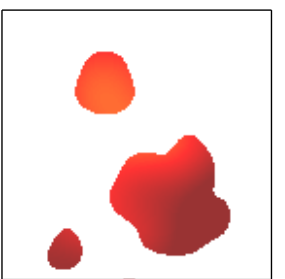

(e) $\widehat{\boldsymbol{C}}_{2}$

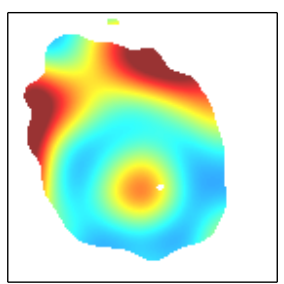

(c) $\widehat{\boldsymbol{W}}_{1}$

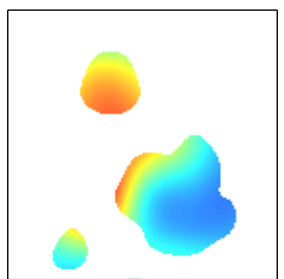

(f) $\widehat{\boldsymbol{W}}_{2}$ $\cdot 10^{-2}$
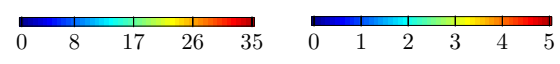

Fig. 5: Real data (galaxy NGC 4254) with $J=2$ structures. (a) Averaged image of the data $\boldsymbol{X}$ along the spectral dimension. (b) Averaged image the reconstruction $\widehat{X}$ (after thresholding) and of the residual (c). (d)-(f) Estimated amplitudes, shifts and shape of the first structure 1. (g)-(i) Estimated amplitudes, shifts and shape of the second structure.

\section{REFERENCES}

[1] D. Mihalas and J. Binney, Galactic astronomy: Structure and kinematics, WH Freeman and Co., 1981. 
[2] E. Villeneuve and H. Carfantan, "Nonlinear deconvolution of hyperspectral data with MCMC for studying the kinematics of galaxies," IEEE Trans. Image Process., vol. 23, 2014.

[3] V. Mazet, C. Collet, and B. Vollmer, "Decomposition and classification of spectral lines in astronomical radio data cubes," in SCIA 2009.

[4] C. Y Peng, L. C. Ho, C. D. Impey, and H.-W. Rix, "Detailed decomposition of galaxy images. II. Beyond axisymmetric models," Astron. J, vol. 139, 2010.

[5] M. Cappellari and E. Emsellem, "Parametric recovery of line-of-sight velocity distributions from absorptionline spectra of galaxies via penalized likelihood," Publ. Astron. Soc. Pac, vol. 116, 2004.

[6] L. Coccato, L. Morelli, E. M. Corsini, L. Buson, A. Pizzella, D. Vergani, and F. Bertola, "Dating the formation of the counter-rotating stellar disc in the spiral galaxy NGC 5719 by disentangling its stellar populations," Mon. Notices Royal Astron. Soc., vol. 412, 2011.

[7] N. Bouché, H. Carfantan, I. Schroetter, L. Michel, D., and T. Contini, "Galpak3d: A Bayesian parametric tool for extracting morphokinematics of galaxies from $3 \mathrm{~d}$ data," Astron. J, vol. 150.

[8] M. Fauvel, Y. Tarabalka, J. A. Benediktsson, J. Chanussot, and J. C. Tilton, "Advances in spectral-spatial classification of hyperspectral images," Proc. IEEE, vol. 101, 2013.

[9] F. Flitti, C. Collet, B. Vollmer, and F. Bonnarel, "Multiband segmentation of a spectroscopic line data cube: Application to the HI data cube of the spiral galaxy NGC 4254," EURASIP J Adv Signal Process, vol. 15, 2005.

[10] P. Comon and C. Jutten, Handbook of Blind Source Separation: Independent Component Analysis and Applications, Academic Press, 2010.

[11] S. Hong and R. A. Harshman, "Shifted factor analysis Part II: Algorithms," J. Chemometrics, vol. 17, 2003.

[12] M. Mörup, K. H. Madsen, and L. K. Hansen, "Shifted non-negative matrix factorization," in IEEE MLSP, 2007.

[13] H. Mortada, V. Mazet, C. Soussen, and C. Collet, "Separation of delayed parameterized sources," in EUSIPCO, 2017.

[14] J. Bobin, J. Rapin, A. Larue, and J.-L. Starck, "Sparsity and adaptivity for the blind separation of partially correlated sources," IEEE Trans. Signal Process., vol. 63, 2015.
[15] C. De Boor, A practical guide to splines, vol. 27, Springer, 1978.

[16] M. Unser, "Splines: A perfect fit for signal and image processing," IEEE Signal Process Mag, vol. 16, 1999.

[17] J. Nocedal and S. J. Wright, Sequential quadratic programming, Springer, 2006.

[18] C. De Boor, "On calculating with B-splines," J. Approx. Theory, 1972.

[19] B. Phookun, S. N. Vogel, and L. G. Mundy, "NGC 4254: a spiral galaxy with an $\mathrm{m}=1$ mode and infalling gas," Astrophys. J, vol. 418, pp. 113, 1993. 